\title{
Screening of Non Tuberculous Mycobacterium Species from Farm Environment
}

\author{
Sangavi Chokkalingam ${ }^{1}$, Porteen Kannan ${ }^{1 *}$, Sureshkannan Sundaram ${ }^{1}$ and B.S. MRonald ${ }^{2}$ \\ ${ }^{1}$ Department of Veterinary Public Health and Epidemiology, Madras Veterinary College, Chennai, Tamil Nadu, INDIA \\ ${ }^{2}$ Department of Veterinary Microbiology, Madras Veterinary College, Chennai, Tamil Nadu, INDIA \\ "Corresponding author: K Porteen; E-mail: rajavet2002@gmail.com
}

Received: 22 March, 2021

Revised: 20 May, 2021

Accepted: 24 May, 2021

\begin{abstract}
The ubiquitous presence of NTM species in the environment is one factor which leads to misdiagnosis, treatment failure of tuberculosis throughout the world. The aim of the present study was to screen samples from farm environment to detect the occurrence of NTM species and to analyze their antimicrobial resistance pattern. A total of 218 samples of water, soil, milk, faeces and sewage were collected and processed as per standard isolation protocols for NTM species. Further, the isolates were subjected to biochemical characterization and molecular confirmation was done by PCR targeting genus specific primers like 16SrRNA and $h s p$ 65. For species identification, the PCR products were sequenced and assessed the genetic relatedness. Antimicrobial resistance of the isolates was also studied by broth microdilution and disc diffusion method. The present study showed the presence of NTM species from farm settings and the isolates were found to be sensitive to antimycobacterial drugs.
\end{abstract}

\section{HIGHLIGHTS}

( M. chelonae and M. abscessus were identified from farm settings.

(0 Sensitivity to first line antimycobacterial drugs

Keywords: Non Tuberculous Mycobacteria, Acid Fast Staining, 16SrRNA, hsp 65, Antimicrobial resistance.

Non Tuberculous Mycobacteria are opportunistic pathogens, causing mostly TB like pulmonary diseases largely in immunocompromised patients or with pre existing disease conditions and extra pulmonary diseases. NTM incidence is highly underestimated because of similar diagnostic patterns with Mycobacterium tuberculosis and Mycobacterium bovis. NTM represents more than 160 species which are commonly found in soil and water systems with varying degree of virulence leading to diverse clinical features. From many studies it is evident that NTM species are isolated from domestic and wild animals, pigs, domestic fowls, captive, exotic and wild birds, their excretions including faeces and also from bedding materials. The presence of NTM is documented from perishable foods and vegetables. Human to human transmission of NTM was not reported till date (Sharma et al., 2020). NTM infections can be detected from the clinical and environmental samples by conventional methods such as acid fast staining, culture in media and based on biochemical parameters. Molecular confirmation is primarily done by PCR targeting various identification markers viz., 16S rRNA, hsp65 and rpoB genes or multi locus sequence typing (MLST) and DNA hybridization techniques. Antimicrobial resistance is also evidenced in NTM organisms. NTM species also share same antimicrobial resistance patterns with the $M$. tuberculosis and $M$. bovis including MDR and XDR. From several studies, it has been noted that NTM species are discrete in susceptibility mechanisms from $M$. tuberculosis. Literature survey has showed the ubiquitous presence of NTM species in various ecosystems. In India, the actual bio burden of NTM species is lacking. The present

How to cite this article: Chokkalingam, S., Kannan, P., Sundaram, S. and MRonald, B.S. (2021). Screening of non tuberculous mycobacterium species from farm environment. J. Anim. Res., 11(3): 467-470. Source of Support: None; Conflict of Interest: None क क 
research was envisioned to study the occurrence of NTM from both organized and unorganized farm environment to identify the most predominant NTM species circulating in the study area and to identify the antimicrobial pattern among the circulating isolates.

\section{MATERIALS AND METHODS}

A total of 218 samples from livestock and farm environment $(\mathrm{n}=218)$ including water $(50)$, soil $(50)$, milk (55), faeces or dung (50) and sewage (13) were collected in a sterile container under aseptic conditions and transported to laboratory under refrigerated conditions and further processed as per Yajko et al. (1995). The samples were subjected to decontamination protocols using $5 \%$ oxalic acid $-4 \% \mathrm{NaOH}$ and $1 \% \mathrm{CPC}$ and incubated for 30 minutes and 24 hours respectively at $37^{\circ} \mathrm{C}$, along with VAN supplement. Decontaminated samples were subjected to acid fast staining and culture isolation was done using Herrold's Egg yolk media and Middlebrook 7H9 broth and subsequently cultured on MB 7H10 agar. The presumptive colonies were subjected to biochemical characterization viz. growth on MacConkey agar, growth in $5 \% \mathrm{NaCl}$, iron re-uptake from the medium, catalase test and Thiophen -2 Carboxylic Hydrazide test. The presumptive NTM isolates were then confirmed by Polymerase Chain Reaction targeting 16SrRNA and $h s p$ 65 genes and the PCR products from the 16SrRNA was sequenced. NTM isolates obtained in the study were subjected to Antimicrobial testing by broth microdilution method for the first line antimycobacterial drugs like rifampicin, isoniazid, ethambutol and pyrazinamide. Disc diffusion method was carried out against amikacin, azithromycin, cotrimaxazole, ciprofloxacin, enrofloxacin, imipenam, doxycycline and rifampicin and the sensitivity pattern and resistance pattern were recorded.

\section{RESULTS AND DISCUSSION}

In this study, decontamination protocols were done with all the 218 samples by utilizing both $5 \%$ Oxalic Acid $4 \% \mathrm{NaOH}$ method and $1 \% \mathrm{CPC}$. Of the two methods, growth was noticed only in samples decontaminated by CPC whereas no growth was noticed in samples decontaminated by Oxalic acid $-\mathrm{NaOH}$. Our study has clearly indicated that $\mathrm{CPC}$ is an efficient decontaminant in the process of culturing NTM species along with VAN supplement. Our results are in accordance with the results of Pardini et al. (2005) who have also found that $0.5 \%$ CPC decontaminated samples can be preserved for longer time and it reduced higher number of contaminants with positive cultures than $1 \% \mathrm{NaCl}$ with NALC $(1 \% \mathrm{NaOH})$.

After decontamination, all the samples were screened for Acid Fast Bacilli by Acid Fast Staining. Of the 218 samples only 6 samples ( 2 water samples, 1 milk sample, 1 soil sample, 2 faecal or dung sample) were found to be harbouring Acid Fast Bacilli. All the 6 samples were further propagated in Herrold's egg yolk media and Middlebrook $7 \mathrm{H} 9$ broth. In this study, we could notice no growth with Herrold's egg yolk media and growth was noticed only in Middlebrook $7 \mathrm{H} 9$ broth and 4 samples were positive which were named M1, S1, W1 and W2. In Middlebrook $7 \mathrm{H} 9$ broth, variation in growth pattern, incubation period and pigmentation was noticed with the isolates. Turbidity without pigmentation was visualized within 5 days in milk, soil and one of the water samples whereas in another water sample, turbidity was noticed with pigmentation within 14 days of incubation. Our result has documented that isolates obtained in the present study viz. water sample (W1), milk sample (M1) and soil sample (S1) were showing turbidity, growth within 5 days and there was absence of pigmentation. Based on this criterion, we could classify our isolates as non chromogenic NTM species which falls under RGM (Bhalla et al., 2018). On the other hand, water sample (W2) showed growth, turbidity and reddish orange color pigmentation over an incubation period of 14 days. Based on this parameter, we could classify our isolate as chromogenic NTM species which falls under SGM.

Samples which showed growth in liquid media were subcultured in MB 7H10 agar. Growth of white buffy colonies in the samples W1, M1 and S1 was indicative of non chromogenic RGM and the lemon yellow colonies in the sample W2 was of chromogenic SGM based on the incubation period. Subculturing in MB $7 \mathrm{H} 10$ agar also suggested that the species might be of $M$. fortuitum, $M$. abscessus and M.chelonae in RGM and some chromogenic SGM. The present study correlates with the study of Ahmed et al. (2013) in which both RGM and SGM were isolated subculturing on MB 7H10 agar.

Biochemical characterization with the isolates obtained in the study yielded varying results. All the 4 isolates were subjected to various biochemical test parameters and the 


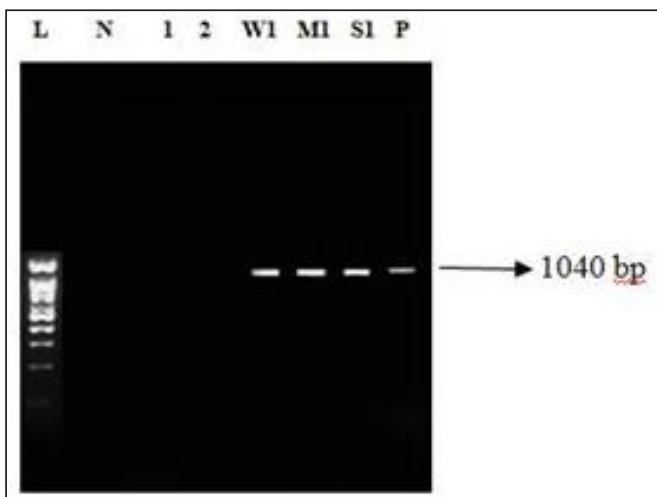

$$
\begin{array}{ll}
\mathrm{L} & -100 \text { bp ladder } \\
\mathrm{N} & - \text { Negative control } \\
1 & - \text { Negative sample } \\
2 & \text { - Negative sample } \\
\mathrm{W} 1 & \text { - Water sample } \\
\mathrm{M} 1 & - \text { Milk sample } \\
\mathrm{S} 1 & \text { - Soil sample } \\
\mathrm{P} & \text { - Positive control }
\end{array}
$$

Fig.1: PCR assay targeting $16 \operatorname{Sr} R N A$ gene

results were correlated with Bhalla et al. (2018) who have described the various methods for distinguishing RGM and SGM species based on biochemical test. With regard to NTM species identification by biochemical tests, it has been reported that it cannot be reliable as it leads to inconclusive results and some of the isolates could not be identified by biochemical tests (Narang et al., 2015). Based on the conventional tests it was noticed that the occurrence of NTM in water, milk and soil were recorded as $4 \%, 1.8 \%$ and $2 \%$ respectively.

Molecular confirmation was carried out by PCR assay targeting 16SrRNA and hsp 65 which revealed that all the 4 isolates were confirmed as Mycobacterium spp. with an amplicon of $1040 \mathrm{bp}$ for 16SrRNA (Fig. 1) and $441 \mathrm{bp}$ for hsp 65 (Fig. 2). Results of our PCR assay targeting 16SrRNA and hsp 65 confirmed the presence of Mycobacterium $\mathrm{sp}$ in water, milk and soil with a percent positivity of $4 \%, 1.8 \%$ and $2.0 \%$ respectively. Results of our PCR assay confirmed all the isolates as positive for Mycobacterium spp. which has already shown growth and tested for biochemical tests is an indication of high sensitivity of the employed PCR assay and it clearly indicated that the assay could be used for routine screening which will aid in the identification of NTM organisms. The PCR products obtained by targeting 16SrRNA were sequenced, assembled, annotated and

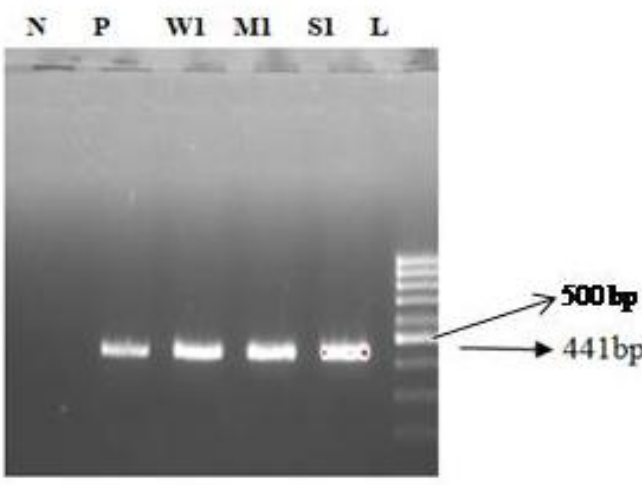

L $\quad-100$ bp ladder

$\mathrm{N}$ - Negative control

W1 - Water sample

M1 - Milk sample

S1 - Soil sample

$\mathrm{P}$ - Positive control
BLAST analysed at NCBI. The milk and soil isolate was identified as $M$. chelonae and $M$. abscessus respectively and the two water samples were identified as uncultured bacteria which is a common phenomena in environmental samples. Our study was in agreement with Sonia et al. (2014) in which they isolated M. Chelonae in milk and with the results of Thomson et al. (2013) and Moore et al. (2020) in which they isolated M. abscessus from water samples. Antimicrobial study by broth microdilution method revealed all the isolates were sensitive to all the first line antimycobacterial drugs. Disc diffusion method showed that $M$. chelonae was resistant to iminepam and intermediately resistant to azithromycin but sensitive to all the other drugs. M. abscessus was resistant to amikacin, arithromycin and intermittently resistant to imipenam and ciprofloxacin but sensitive to all the other drugs.

\section{CONCLUSION}

This study identified the occurrence of NTM species like M. chelonae and M. abscessus from milk and soil samples is an indication of presence of these organisms in farm settings. Considering the potential of this pathogen in relation to human and animal infections there is a need for devising appropriate control strategies to prevent the 
transmission and occurrence at farm level and to safeguard the health of farm workers, general public with special concern to immuno compromised individuals.

\section{ACKNOWLEDGEMENTS}

The authors are thankful to TANUVAS, for financial support during the study.

\section{REFERENCES}

Ahmed, I., Jabeen, K. and Hasan, R. 2013. Identification of nontuberculous mycobacteria isolated from clinical specimens at a tertiary care hospital: a cross-sectional study. J. Infect. Dis., 13: 493.

Bhalla, G.S., Sarao, M.S., Kalra, D., Bandyopadhyay, K. and John, A.R. 2018. Methods of phenotypic identification of non-tuberculous mycobacteria. Pract. Lab. Med., 12: e00107.

Biet, F. and Boschiroli, M.L. 2014. Non-tuberculous mycobacterial infections of veterinary relevance. Res. Vet. Sci., 97: S69-S77.

Covert, T.C., Rodgers, M.R., Reyes, A.L. and Stema, G.N. 1999. Occurrence of Nontuberculous mycobacteria in environmental sample. Appl. Environ. Microbiol., 65: 24922496.

Falkinham III, J. O., 2002. Nontuberculous mycobacteria in the environment. Clin. Chest. Med., 23: 529- 551.

Ichiyama, S., Shimokata, K. and Tsukumara, M. 1988. The Isolation of Mycobacterium avium Complex from Soil, Water, and Dusts. Microbiol. Immunol., 32 (7): 733-739.

Jesudason, M.V. and Gladstone, P. 2005. Non Tuberculous Mycobacteria isolated from clinical Specimens at a tertiary care hospital in South India. Indian J. Med. Microbiol., 23(3): $172-175$

Kamala, T., Paramasivan, C.N., Herbert, D., Venkatesan, P. and Prabhakar, R. 1994. Isolation and identiccation of environmental mycobacteria in the Mycobacterium bovis BCG trial area of South- India. Appl. Environ. Microbiol., 60: $2180-2183$.

Mendum T.A., Chilima B.Z. and Hirsch, P.R. 2000. The PCR amplification of non-tuberculous mycobacterial 16S rRNA sequences from soil. FEMS Microbiol. Lett., 185: 189-192.
Moore, J.E., Moore, R.E., Shteinberg, M., Kis-Papo, T. and Millar, B.C. 2020. Survival of Mycobacterium abscessus and Staphylococcus aureus in saline waters of the Dead Sea: implications for health tourists. J. Travel. Med., 27(6): taaa089.

Narang, P., Mendiratta, D.K., Tyagi, N.K., Jajoo, U.N., Tayade, A.T., Parihar, P.H., Narang, R., Mishra, P.S., Anjinappa, S.M. and Khadha, V. 2015. Prevalence of pulmonary tuberculosis in Wardha district of Maharashtra, Central India. J. Epidemiol. Global Health., 5(4): S11-S18.

Pardini, M., Varaine, F., Iona, E., Arzumanian, E., Checchi, F., Oggioni, M. R., Oreficiand, G., and Fattorini, L. 2005. CetylPyridinium Chloride Is Useful for Isolation of Mycobacterium tuberculosis from Sputa Subjected to Long-Term Storage. J. Clin. Microbiol., 43(1): 442-444.

Sharma, K., Menia, N., Bajgai, P., Sharma, M., Sharma, A., Katoch, D. and Singh, R. 2020. Nontubercular mycobacteria associated uveitis in HIV positive patients. Ocul. Immunol. Inflam., : 1-8

Somoskovi, A., Mester, J., Halee, Y.M., Parsonsd, L.M. and Salfinger, M.D.M. 2002. Laboratory diagnosis of nontuberculous mycobacteria. Clin. Chest. Med., 23: 585597.

Sônia, A.S., Hirata, R.D.C., Hirata, M.H., Leite, C.Q.F., de Prince, K.A., de A. Leite, S.R., Filho, D.V., Siqueira, V.L. D., Ferracioli K. R.C. and Cardoso, R.F. 2014. Occurrence of Mycobacterium bovis and non-tuberculous mycobacteria (NTM) in raw and pasteurized milk in the northwestern region of Paraná, Brazil. Brazilian J. Microbiol., 45(2): 707711.

Thomson, R., Tolson, C., Sidjabat, H., Huygens F., and Hargreaves, M. 2013. Mycobacterium abscessus isolated from municipal water - a potential source of human infection. BMC Infect. Dis., 13: 241

Williams, D.L., Gillis, T.P. and Booth, R.J. 1990. The use of a specific DNA probe and polymerase chain reaction for the detection of Mycobucterium Ieprae. J. Infect Dis., 162: 193200

Yajko, D.M., Chin, D.P., Gonzalez, P.C., Nassos, P.S., Hopewell, P.C., Reingold, A.L., Horsburgh, C.R., Yakrus, M.A. and Ostreo;, S.M. 1995. Mycobacterium avium complex in water, food and soil samples collected from the environment of HIV-infected individuals. J. Acquired Immun. Decc. Synd. Hum. Retrovirol., 9: 176-18 